\title{
Eliciting Goals for Business Process Models with Non-Functional Requirements Catalogues
}

\author{
Evellin C. S. Cardoso, João Paulo A. Almeida, \\ Giancarlo Guizzardi, Renata S. S. Guizzardi \\ Ontologies and Conceptual Modelling Research Group (NEMO) \\ Computer Science Department, Federal University of Espírito Santo (UFES), \\ Av. Fernando Ferrari, s/n, Vitória, ES, Brazil \\ ecardoso@inf.ufes.br,jpalmeida@ieee.org,gguizzardi@acm.org,rguizzardi@inf.ufes.br
}

\begin{abstract}
While traditional approaches in business process modelling tend to focus on "how" the business processes are performed (adopting a behavioural description in which business processes are described in terms of procedural aspects), in goal-oriented business process modelling [23][24][6], the proposals strive to extend traditional business process methodologies by providing a dimension of intentionality to the business processes. One of the difficulties in enabling goal-oriented business process modelling is the identification of goals. This paper reports on a study conducted in an organization in which we have obtained several goal models which were represented in Tropos methodology, each one corresponding to a business process also modelled in the scope of the study. A preliminary goal elicitation activity has been carried out for collecting an initial version of the goal models. After that, we have obtained a second version of the goal models by using the NFR catalogues as a tool in goal elicitation. We have found the NFR catalogues to be useful in goal elicitation, uncovering goals that did not arise during previous interviews.
\end{abstract}

Keywords: business processes, goal models, non-functional requirements

\section{Introduction}

The increasing competitiveness drives organizations to promote change in an attempt to improve the quality of the services and products they offer. In recent years, many of the efforts related to managing change in organizations have been conducted in the scope of Business Process Reengineering (BPR) activities [1][2]. BPR is based on the assumption that change in business processes should generate radical improvements in critical performance measures (such as cost, quality, service and speed) [1]. Moreover, it is believed that implementing radical changes in business processes is the way to achieve dramatic and satisfactory results [1][2].

However, predicting how a given enterprise environment should respond to changes by simply adopting a business-process centered view is unfeasible since there is a large number of issues to be considered, such as infrastructure, power and politics, organizational culture, etc [3]. Given this multitude of issues, understanding an organizational setting often requires a number of perspectives [3]. 
While traditional approaches in business process modelling tend to focus on "how" the business processes are performed (adopting a behavioural description in which business processes are described in terms of procedural aspects), in goal-oriented business process modelling [23][24][6], the proposals strive to extend traditional business process methodologies by providing a dimension of intentionality to the business processes [4].

In recent years, goal-oriented approaches have been largely addressed in the literature of requirements engineering (RE) which focuses on how these approaches support requirements elicitation and modelling for system development [4]. In this context, goals express, in multiple levels of abstraction, the objectives the system under consideration should undertake [8]. Although this field is mainly concerned with system development, a goal-driven nature of software engineering requires RE to pay attention to the organizational context [4]. The Zachman framework [22] also highlights the importance of motivation as a driver for enterprise management and system development. Therefore, in the context of business process modelling, goal modelling is extended not only to capture concerns and motivations of the stakeholders in the achievement of business processes, but to incorporate issues related with the strategy of the enterprise as a whole.

Although goal modelling provides a more strategic view for business processes, little attention is devoted to explicitly modelling goals as well as using the concept of goal to increase the value of the process modelling techniques [12]. To articulate an organization's business processes in terms of the enterprise's strategic goals, the first problem to be addressed is how to elicite goals from the organization context since goal identification is not an easy task [8]. Problems related with goal elicitation are firstly addressed by the requirements engineering (RE) literature, but essentially the same problems arise in the area of business process modelling. Some problems arising in the scope of business process modelling are: goals are difficult to formulate (often these formulations become vague and highly abstract) [7], the involved parties are unable to explicitly state their views [14] and even when they are capable the goals can be conflicting [15] (even when goals are drawn from the same individual), the analysts have limited knowledge about the environment [14], among others.

The purpose of this paper is to report a field work in which we addressed the problem of identifying goals. This field work took place in the Rheumatology Department of a hospital in Brazil. Our main purpose is to contribute in the area of goal elicitation with a systematic way to identify goals in a given organizational setting in the business process modelling activity prior to the identification of potential business process change. We suggest using Non-Functional Requirements (NFRs) [9][10] catalogues in order to tackle the difficulty in identifying business goals. The next section shows our motivation in adopting this approach.

This paper is structured as follows: section 2 describes the NFR framework (used as a starting point to derive (soft)goals), section 3 provides a description of our field work: the preliminary goal elicitation method (section 3.1), the results of this preliminary phase (section 3.2), the goal elicitation with catalogues (section 3.3), a discussion about these results (section 3.4). Section 4 points to related work and finally, section 5 concludes with some considerations as well as future research work. 


\section{The NFR framework}

Many important goals for an organization may be characterized as "softgoals", i.e., goals with no clear-cut criteria to determine their satisfaction [5]. Eliciting softgoals is particularly challenging, given their subjective, interactive and relative nature [16]. One of the solutions proposed to address the problem of identifying non-functional requirements in RE is the NFR framework. Basically, the NFR framework is an approach for specifying and analyzing non-functional requirements (NFRs). Although this framework has been primarily conceived for helping one to elicit NFRs in the system development life-cycle, we have used them to tackle the problem of systematic identifying business goals, helping one to reflect about the dimensions which a softgoal can assume in an organizational environment.

Although the concepts of NFRs and softgoals are commonly treated as the same in the literature, we must stress out that the differences between NFRs and softgoals cannot be ignored. On one hand, as explained in [25], NFRs refer to quality attributes that some system is expect to meet while executing a particular service. These services which the system must provide amounts to its set of functional requirements (FRs). NFRs are the opposite of functional requirements (FRs). On the other hand, hardgoals are defined as goals whose satisfaction can be determined by applying formal verification techniques [8][25]. Softgoals are the opposite of hardgoals, since they are "subject to interpretation" [3], "imprecise, subjective, context-specific, and ideal" [25]. This common association between NFRs and softgoals arises because there is a tendency in specifying quality attributes in an imprecise manner.

Since we have addressed the motivation behind using the NFR framework and the difference between NFRs and softgoals, we describe the NFR framework in the sequel.

The NFR Framework [9][10][16] comprises a proposal for addressing NFRs using catalogues that accumulates knowledge about specifying NFRs. The catalogues' specification ranges from representing to operationalizing NFRs, offering guidelines for prioritization and decomposition during the design process.

The Softgoal Interdependency Graphs (SIGs) represent particular kinds of NFRs, their decomposition structures and possible design alternatives to embody the requirement in the future system. Further, the interdependencies between the NFRs and their operationalizations are represented.

There are three kinds of catalogs used: the first kind (called NFR type catalog) contains particular types of NFRs, such as security or performance, and their associated terminology. The second type (called methods catalogs) represents development techniques for the system to meet a particular requirement and finally, the third type (correlation catalogs) shows the correlation and tradeoffs among softgoals.

Fig. 1 depicts a catalogue of some NFR types, where a NFR can be "performance", "security", "cost" and "user-friendliness" [9]. 


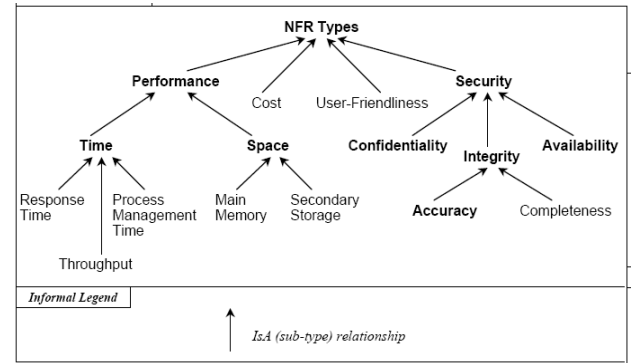

Fig. 1. A catalogue of some NFR types [9]

An application within a real example is also demonstrated in [9]. In this example, security is the NFR considered for developing a credit card system. Fig. 2 shows that to incorporate security in a given account, three subtypes of NFRs are necessary: integrity, confidentiality and availability. In turn, to incorporate integrity on credit card accounts, two additional NFRs are needed: completeness and accuracy. This example depicts that the process of decomposing some NFR is guided (and thus facilitated) by adopting these catalogues since they are helpful in reasoning about what qualities the system to-be is expected to meet.

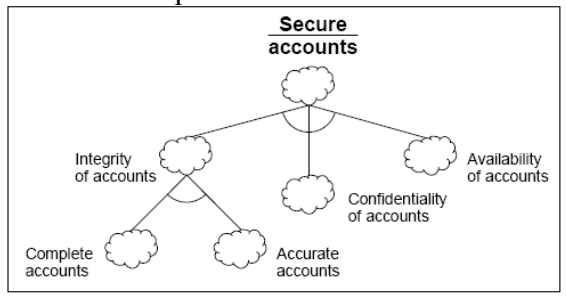

Fig. 2 Further decomposition of a security softgoal (adapted from [9])

\section{Case Study: Goal Elicitation in a Healthcare Institution}

This section describes the case study conducted in the Rheumatology Department of the Cassiano de Moraes University Hospital, which is part of the Federal University of Espírito Santo in Vitória, Brazil.

In the context of the hospital, the department has the following functions: provide educational training to form specialists in rheumatology, provide outpatient medical care and developing research to investigate the incidence de rheumatologic conditions in population. It has six specialists in rheumatology, two nurses and two physiotherapists, among other professionals for hosting patients. The department performs 15 business processes, such as outpatient care, drugs infusion, among others and performs an average rate of 5700 outpatient medical care by year.

In the scope of this study, we have produced 9 goal models which were represented using the Tropos Modelling Language and methodology [17]. Each of these models correspond to a business process also modelled in the scope of the study. A 
preliminary goal elicitation activity has been carried out for collecting an initial version of the goal models. After that, we have obtained a second version of the goal models by using the NFR catalogues as a tool in goal elicitation. Due to space restrictions, we focus on the goal models of the diagnosis process.

\subsection{Preliminary Goal Elicitation Method}

This preliminary goal elicitation and modelling effort was divided in four stages according to the source of information and technique used to interact with the process stakeholders. In the first and second stages, we have captured only hardgoals. From the second stage on, our goal models were composed by hardgoals and softgoals.

In a first stage, the available documentation about the organizational process has been assessed. This revealed some organizational characteristics such as: organizational structure and human resources, routines, business processes (with a brief textual explanation in natural language about these processes) and physical space. From the organization structure, we could infer internal actors and the business process they carry out. This documentation also provided goals previously achieved by the department (along with their impacts) and goals which were yet to be achieved by the department, giving us some insight about the nature of the business processes under consideration and about some relevant goals (stated in natural language). Further, a first interview was undertaken with a physician (who does not belong to the organization) for understanding general concepts about the medical domain. Additionally, concepts related with rheumatology (diseases, medicines and other technical terms) have been briefly surveyed in online information sources.

In a second stage, we have obtained a preliminary goal model along with a preliminary business process model. The approach used here consisted in observing the process performers during business process execution, i.e., we observed the daily routine of the organization. While this approach allowed us to understand how actors interact and how actor dependency relationships are established in practice, the actors' focus on getting the work done prevents one from revealing most of the intention and motivation behind their practices.

A third stage focused on interviewing the organizational actors during business process execution to reveal the goals of specific activities as well as goals related with a process as a whole. Thus, the model generated in previous stage could be incremented through refinement/abstraction techniques [3][8][18]. With the refinement technique one can find out subgoals of the parent goal by asking "HOW questions" about the goals already identified [8]. This is helpful in capturing the different ways of goal achievement. With the abstraction technique, more abstract goals can be identified by asking "WHY questions" about the goals previously modelled [8]. This enabled us to capture the rationale (more general goals) behind more specific goals. Although the interviews during the process execution provided a more strategic dimension (in the sense that they have captured details related with the organization's strategy in a lower level of abstraction), the goal models obtained were strongly related to the business process models, not capturing knowledge about the enterprise setting as a whole. This deficiency was addressed in fourth stage.

In a fourth stage, we concentrated in dedicated interviews not only with the business process actors but also with the department manager. (By "dedicated interviews" we mean that the interviewees devoted all attention to the elicitation 
process as opposed to being fully involved with activity execution). The elicitation interviews in this stage focused on raising internal problems of the organization, as well as problems associated with the relationship between the department and external organizations, highlighting all kinds of conflicting interests. The problems and deficiencies that the stakeholders believed to exist in the organization provided not just additional goals to enrich the models, but also some obstacles for goal realization, reasons for non-achievement of goals and possible solutions for these obstacles.

\subsection{Results of the Preliminary Goal Elicitation Activities}

Fig. 3 exhibits a Tropos diagram which shows the goals of a physician who conducts the diagnosis business process.

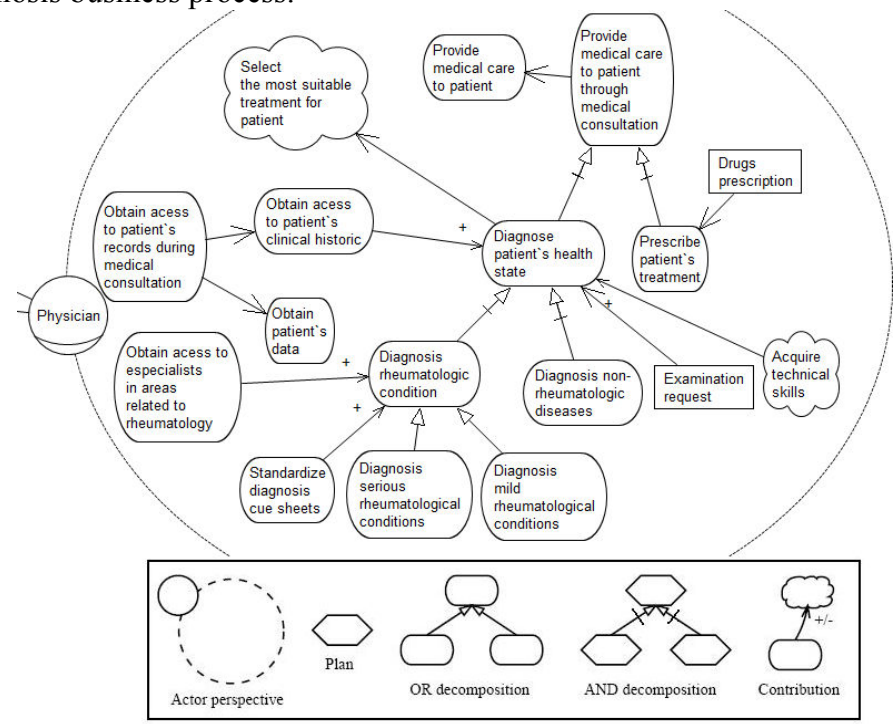

Fig. 3. Goal model resulted from the preliminary goal elicitation activities

In Tropos diagrams, actors are represented as circles, goals as oval shapes and softgoals as cloud shapes. (Soft)goals can be related with three kinds of relationships: means-end analysis, contribution analysis and AND/OR decomposition. ${ }^{1}$

The physician provides medical care to a patient (Provide medical care to patient goal) through a medical consultation (Provide medical care through medical consultation goal). During consultation, the physician diagnoses the patient's health state (Diagnose health state goal) and prescribes the treatment (Prescribe patient's treatment goal which uses, in turn, a Drugs prescription).

The main goal of the physician is to Diagnose patient's health state. During the process of diagnosis, the physician can find either rheumatologic or nonrheumatologic conditions (Diagnose rheumatologic conditions goal and Diagnose non-rheumatologic conditions goal). After diagnosing the patient's heath state, the

${ }^{11}$ The AND/OR decomposition can be made by using the "HOW questions" previously mentioned. 
physician is able to select the most suitable treatment for the disease (Select the most suitable treatment for patient softgoal). For this reason, Diagnose patient's health state is a mean for Select the most suitable treatment for patient.

The physician must have accurate knowledge for being able to discover the presence/absence of diseases (Acquire technical skills softgoal). He/she must also access the patient's data (Obtain patient's data goal) for being able to determine how the patient health condition is evolving along the time (Obtain access to patient's clinical historic goal). One of the means for accessing the patient's data and thus to know its clinical history is to obtaining access to patient's records (Obtain access to patient's records during medical consultation goal). Finally, the rheumatologist must confirm the diagnosis with other specialists in order to interpret the evidences in the whole clinical context (Obtain access to specialists in areas related to rheumatology goal).

\subsection{Goal Elicitation with Catalogues}

During the four stages we have reported here, we had the opportunity to understand the organization's context, its problems, deficiencies and so forth. By following the execution of the business process, interviewing the stakeholders and observing the organizational setting, we could keep direct contact with implicit factors that underlie the organizational context. These previous stages were crucial to provide insights about new concerns that could be added. These insights guided us to suggest which NFR types could be extracted from NFR catalogues [9][10][19][20] and subsequently adapted to the organizational context. According the NFR types catalogues, we have formulated additional goals for the business process, initially without participation of the stakeholders. The translation from NFR types in the catalogues to goals was highly related to the knowledge acquired in previous stages, i.e., to adequately refine the NFRs we had to consider the meaning of the NFRs' refinement in the context of the domain under consideration. After incorporating these additional goals into the model, we have applied the same techniques of abstraction/refinement previously applied for identifying additional goals without the participation of the stakeholders. Due to space constraints, we concentrate here on some relevant portions of the resulting goal models.

\subsection{Results of the Goal Elicitation with Catalogues}

Before discussing the outcomes related with the utilization of the catalogues with the stakeholders, we have translated the NFR types to goals in the context of the domain under consideration. The NFR types originated the following goals:

Accessibility [19]. Access patient's data records;

Confidentiality [19]. Maintain healthcare information private;

Completeness [19]. Obtain complete information about patient's treatment;

Accuracy [19]. Obtain accurate information about patient's treatment;

Traceability (process and data) [19][10]. Obtain traceability for information in patient's treatment refined into Obtain traceability in investigation of patient's condition, Obtain traceability in relation to treatment administered to patient and Obtain traceability in relation to physicians who prescribed patient's treatment. 
Integrability [19]. Integrate service with other hospital departments, Integrate service with municipal and state health services (to obtain what is called "integrated treatment" exploring the benefits of information integration).

Trust and confidence to the provider (assurance) [20]. Trust physician (not shown in the figures since this goal belongs to the patient's perspective.)

Empathy (level of caring and personalized attention provided to the requestor) [20]. Show empathy to patient.

The translation of NFR types from the catalogues to goals revealed that although catalogues address non-functional requirements, some goals elicited in this effort are not softgoals, but hardgoals instead. For instance, the requirement of Accessibility has led to the identification of the hardgoal Access patient's records. Besides, the translation seems to be highly domain-dependent. For example, traceability refers to the capacity of tracing patient's data along treatment. Another particularity concerned with the translation is that different NFR types are mapped to the same goal in the organization. Distributivity (capacity of reaching all decision-makers [19]) and integrability (capacity of adequately and efficiently integrating operational information [19]) mean the same in this context (in the sense that both mean the information must be integrated so as to reach all decision-makers caring about that information). Privacy and confidentiality are also mapped to the same goal.

With respect to the goals added, we were able to identify goals which had remained implicit in the preliminary study (Fig. 4). Most of these goals were either associated with quality aspects of the previously modelled goals (Obtain complete information about patient's treatment softgoal and Obtain accurate information about patient's treatment softgoal) or with quality aspects for the service as a whole (Integrate service with all stakeholders softgoal and the softgoals originated from its refinements). We also have noticed that some of some of the elicited (soft)goals address exceptional situations, for example, the softgoal Integrate services with specialists in areas related to rheumatology is relevant only in the case the rheumatologist needs to clarify further details about the diagnosis with other specialists (for example, a dermatologist or ophthalmologist) in the hospital.

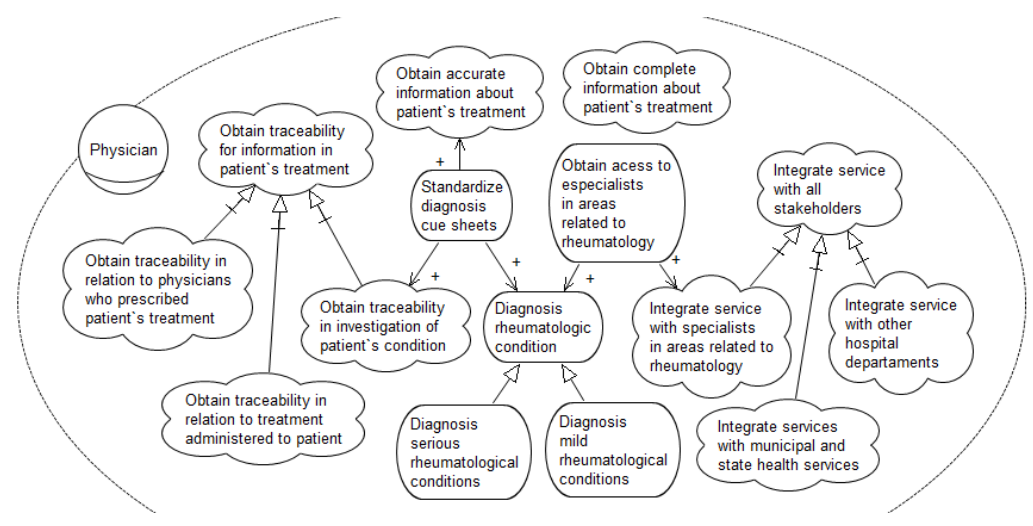

Fig. 4 Portion of the goal model obtained in goal elicitation activities with catalogues (1) 
Another interesting aspect in elicitation with catalogues was the fact that we could notice that some of the goals spontaneously mentioned are actually goals for implementing mechanisms for the attainment of more abstract goals, which remained implicit when applying the abstraction technique, but that could be revealed through the use of the catalogues. For instance, in Fig. 4, from traceability we have suggested three types of traceability: Obtain traceability in relation to treatment administered to patient softgoal (obtain information about the drugs prescribed along the treatment), Obtain traceability in relation to physicians who prescribed patient's treatment softgoal (obtain information about the physicians who had already prescribed treatment to the patient) and Obtain traceability in investigation of patient's condition softgoal (obtain information about the conditions which had already been investigated previously by the physician). Actually, this last goal was the motivation for the standardization of diagnosis cue sheets (previously modelled). The standardization of diagnosis cue sheets was one of many means towards achieving traceability in the investigation of diseases.

Finally, all goals suggested through the use of catalogues were validated by the stakeholders in a validation interview. They acknowledged the need of these goals and were also able to spontaneously mention other goals (for example the refinements of the goal Provide medical care to patient goal, shown in Fig. 5). The goal Provide medical care to patient can be achieved in three forms: by achieving a consultation appointment (in this consultation the physician examines the patient and prescribes the treatment); by providing attendance for assessment of high cost drug (the physician examines the patient and in the case of the need of a high cost drug, he/she issues an certificate) and by an informal meeting. In these informal meetings, the physician can examine a patient who reports the presence of symptoms, or the physician just issues some document required by the patient (a prescription of drugs, a medical certificate or a medical report).

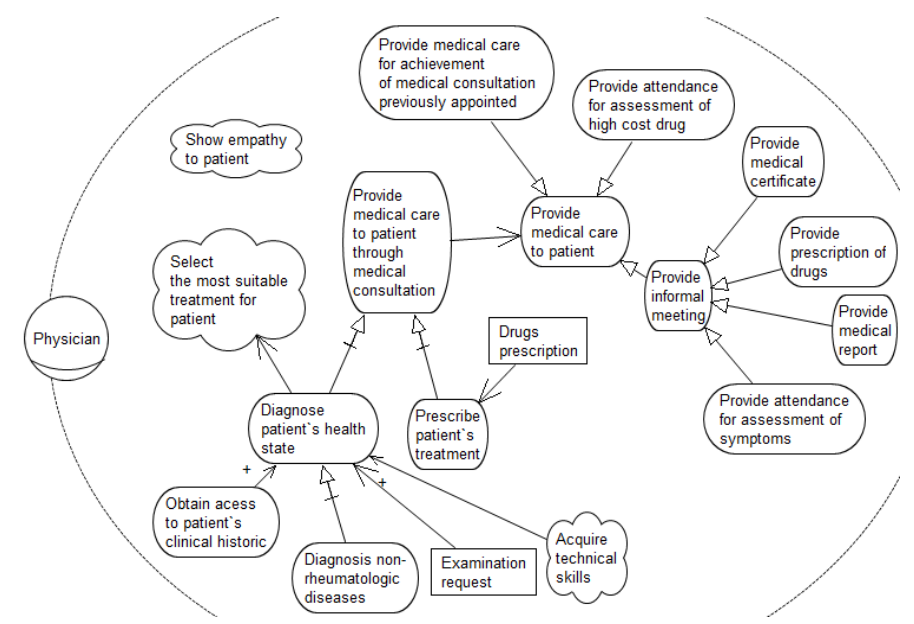

Fig. 5. Portion of goal model obtained in goal elicitation activities with catalogues (2) 


\section{Related Work}

The NFR framework [9] [10] has been the first approach to propose softgoals and softgoal satisfacing in the context of RE to cope with NFRs and their appropriate representation [11]. The main proposal of this framework is to provide means of specifying NFRs, decomposing them and recording the reasoning process of operationalizing and prioritizing NFRs in graphs. Although the catalogues proposed in that work serve as useful tools in identifying softgoals, they are only concerned with addressing these issues in the context of systems development, not considering them within the organizational scope.

In [12], Soffer and Wand propose a conceptual framework to enable the formal integration of goals and softgoals into process modelling (formal in the sense that the approach uses the BWW ontology [13] for the integration). The application of this framework drives the mechanism of systematically reasoning about the incorporation of the softgoals in process design. Although the initiative focuses on defining the existing dependencies between process and their respective softgoals, the method starts in the point where softgoals are already identified (it assumes that the goal elicitation phase has already been undertook) and thus, does not provide guidelines for systematic addressing goal elicitation from the organizational context.

Pavlovski and Zou [21] assume that business process modelling is concerned about capturing functional behaviour of business process but fail to cover non-functional requirements. To tackle this deficiency, the authors propose two new artifacts to capture these constraints denominated operating condition (to represent the business process constraints) and control case (to denote criteria to manage the risk associated with a given operational condition), to be applied in business process models. For that, it extends the Business Process Modelling Notation (BPMN) with these constructs. Although these artifacts have improved the communication about NFRs among the stakeholders, thus facilitated their acquisition, the proposal adopts a system-oriented view for non-functional requirements. It assumes that softgoals will later manifest themselves as non-functional requirements of an intended system, excluding those softgoals which are intrinsically related to the business process' dynamics and, as consequence, which should not be translated into system requirements.

Extending the notion of goals in business process modelling, $\mathrm{Yu}$ [3] has proposed the $i^{*}$ framework. The Tropos methodology has been derived from the $i^{*}$ framework, addressing the need of a methodology for software development based on the agentoriented paradigm. The following explanation about the $i^{*}$ framework can also be applied to the Tropos methodology.

The $i^{*}$ framework is articulated in terms of two kinds of models: the Strategic Dependency model (SD) and Strategic Rationale model (SR). The SD model acknowledges actors as intentional agents within an organizational setting and represents them as a network of strategic dependencies. The SR assumes that the dependencies expressed in SD models are justified by actors' internal rationales. As explained in Yu's $\mathrm{PhD}$ thesis [3], the framework can be used to capture (soft)goals in business process modelling in order to provide a more strategic view of those processes. However, despite the usefulness of the $i^{*}$ approach to address intentions, 
motivations and vague aspects of business modelling, their work do not provide methodological guidelines for eliciting goals.

This paper proposes the use of NFR catalogues for goal elicitation within goaloriented business process modelling in goal models based on Tropos modelling language. This combination the NFR framework and the Tropos modelling language has the following direct benefits. On one hand, it extends the use of NFR framework (initially conceived for addressing NFRs in system development) to support the identification of business goals within organizational scopes. On the other hand, it provides methodological guidelines for the identification of business goals in Tropos. In this manner, the SR and SD Tropos diagrams can be enriched by the additional goals identified through the use of NFR catalogues.

\section{Conclusions}

We have found the preliminary goal elicitation activities useful in addressing our need to understand the organizational setting. This has enabled us not only to capture details about the enterprise and its business processes, but also to provide us with proper understanding about the domain under consideration. However, we have found the preliminary stages to be deficient in the identification of strategic concerns related to the organization's goals. This difficulty was partly addressed through stakeholder interviews. Although these interviews addressed many organizational issues, much knowledge still remained implicit. With respect to that, the catalogues provided by the NFR framework have shown to be useful as a complementary tool to elicit goals.

Before discussing the nature of the additional goals identified with the support of catalogues, we must highlight some particularities about translating NFR types to goals. We have observed that the translation is highly domain-dependent, i.e., one must take into account how a NFR must be mapped to some goal in the organization domain such that this goal makes sense regarding the organizational context, as we have illustrated in section 3.4. Further, one must define whether a NFR type should be represented as a soft or hard goal. As observed in [11], there is a tendency in treating NFRs as softgoals, however, as demonstrated in the case study, some NFRs could be objectively specified in the context of the domain.

In relation to the goals uncovered with the help of catalogues, we believe that goals have enabled us to reason about the organization from a more strategic point of view. This can be confirmed by the fact that some additional goals referred to quality attributes; either for specifying qualitatively a hardgoal or for specifying quality metrics for the business process as a whole. We have observed in this case study that stakeholders have difficulties in explicitly stating quality attributes for business processes (the same difficulty is often reported to arise requirements in system development [16]). In that respect, the catalogues employed in this case study provided guidelines for identifying these attributes in a systematic way.

We have observed that, in certain cases, stakeholders formulate goals which are highly dependent on the current operationalization of the organization's objectives, i.e., much emphasis is given to the goal of applying successfully a particular solution for a problem. Catalogues partially helped to overcome this issue, revealing higher 
level goals not easily identified by the abstraction technique. Further, some of the goals uncovered through catalogues had initially been deemed an inherent organizational characteristic by stakeholders, and thus had not been spontaneously mentioned.

At first sight, the technique we have employed seems highly dependent on the experience of analysts in conducting the elicitation effort (experience in the sense that analysts must have broad knowledge about the domain). We believe this is the case partly because of the need to translate NFRs into goals which are specific to the organization's domain. Further investigation in NFR type catalogues for business process in a particular business domain may prove to be fruitful to reduce the dependency on analyst experience and improve goal elicitation in general. In this sense, NFR type catalogues can be seen as design patterns in goal modelling. The compilation of these catalogues in a format of design patterns allows one to reuse the knowledge by making available methodological connections which are tacit in an experienced modeller's mind and which are not typically available to the novice.

Further work will be necessary to associate particular goals with guidelines for business process (re-)design. Additionally, in our future work, we intend to investigate suitable representation and semantics to relate goal models and business process models (especially in the presence of softgoals). Moreover, we aim at investigating the impact which this approach of eliciting additional goals through the use of NFR catalogues shall have in business process structures as well in the systematic redesign of business processes.

Acknowledgments. This work was partially supported by FAPES in the scope of the INFRA-MODELA project and in the scope of grant DCR number 37274554/2007. We thank to all physicians, interns, residents and patients at the Cassiano de Moraes University Hospital for their cooperation in this research. In particular, we are grateful to Professor Dr. Valéria Valim and Érica Serrano, MD for providing invaluable assistance in the execution of this research in the Hospital.

\section{References}

1. Hammer, M.., Champy, J.: Reengineering the Corporation: A Manifesto for Business Revolution, HarperBusiness (1993)

2. Hammer, M.: Reengineering Work: Don't Automate, Obliterate, Harvard Business Review (1990)

3. Yu, E.: Modelling Strategic Relationships for Process Reengineering, $\mathrm{PhD}$ Thesis, University of Toronto (1995)

4. Kavakli, E., Loucopoulos, P.: Goal Driven Requirements Engineering: Evaluation of Current Methods. In Proceedings of the 8th CAiSE/IFIP8.1 Workshop on Evaluation of Modeling Methods in Systems Analysis and Design, EMMSAD (2003)

5. Mylopoulos, J., Chung, L., Yu, E., Nixon, B.: Representing and Using Non-functional Requirements: A Process-Oriented Approach, IEEE Trans. on Software Eng, 18(6), pp:483497 (1992)

6. Neiger, D., Churilov, L.: Goal-Oriented Business Process Modeling with EPCs and ValueFocused Thinking. Business Process Management (2004) 
7. Halleux, P., Mathieu, L., Andersson, B.: A Method to Support the Alignment of Business Models and Goal Models. In Proc. 3rd Workshop on Business/IT-Alignment and Interoperability (BUSITAL'08) CEUR Workshop Proceedings (2008)

8. Lamsweerde, A.: Goal-Oriented Requirements Engineering: A Guided Tour, 5th International Symposium on Requirements Engineering, IEEE Computer Society Press, (2001)

9. Chung, L., Nixon, B. A., Yu, E., Mylopoulos, J.: Non-functional Requirements in Software Engineering, Kluwer Academic Publishing (2000)

10. Catalogues: http://math.yorku.ca/ cysneiro/nfrs/nfrs.htm, accessed in 19/02/2008

11. Daneva, M., Kassab, M., Ponisio, M.L., Wieringa, R.J., Ormandjieva, O.: Exploiting a Goal Decomposition Technique to Prioritize Non-functional Requirements. In: Proceedings of the 10th International Workshop on Requirements Engineering (WER'07) (2007)

12. Soffer, P., Wand, Y.: On the notion of soft-goals in business process modeling. BPM Journal, pages 663- 679 (2005)

13. Wand, Y., Weber, R.: An ontological model of an information system, IEEE Transactions on Software Engineering, Vol. 16 No. 11, pp. 1282-92 (1990)

14. Dardenne, A., Lamsweerde, A., Fickas, S.: Goal-Directed Requirements Acquisition, Science of Computer Programming, Vol. 20 (1993)

15. Alexander, I.: Modelling the Interplay of Conflicting Goals with Use and Misuse Cases. Proceedings of the $\mathrm{HCI}^{*} 02$ Workshop on Goal-Oriented Business-Process Modeling (GBPM 2002) (2002)

16. Cysneiros, L.M: Evaluating the Effectiveness of using Catalogues to Elicit Non-Functional Requirements. In: Proc. of 10th Workshop in Requirements Engineering, pp 107-115 (2007)

17. Bresciani, P., Perini, A., Giorgini, P., Giunchiglia, F., Mylopoulos, J.: Tropos: An AgentOriented Software Development Methodology. Autonomous Agents and Multi- Agent Systems (2004)

18. Regev, G., Wegmann, A.: Where do Goals Come from: the Underlying Principles of GoalOriented Requirements Engineering. Proceedings. 13th IEEE International Requirements Engineering Conference (2005)

19. Castro, J. F. B., Paim, F. R..: Enhancing Data Warehouse Quality with the NFR Framework. In Proceedings of the V Workshop on Requirements Engineering. Valencia: Universidad Politecnica de Valencia (2002)

20. O'Sullivan, J., Edmond, D., Hofstede, A. T.:What's In a Service? Towards Accurate Description of Non-Functional Service Properties. Distributed and Parallel Databases, 117133, Kluwer Academic Publishers (2002)

21. Pavlovski, C. J. and Zou, J.: Non-functional requirements in business process modeling. In Proceedings of the Fifth on Asia-Pacific Conference on Conceptual Modelling - Volume 79 (2008)

22. Zachman, J.A.: A framework for information systems architecture, IBM Systems Journal, 26 (3), pp. 276-292 (1987)

23. Yamamoto, S., Kaiya, H., Cox, K., Bleistein, S.: Goal Oriented Requirements Engineering: Trends and Issues. IEICE - Trans. Information Systems E89-D, 11 (2006).

24. Proceedings of the HCI*02 Workshop on Goal-Oriented Business Process Modeling GBMP'02 London, September 2, Edited by Ilia Bider and Paul Johannesson (2002)

25. Jureta, I. J., Faulkner, S., Schobbens P. Y.: A More Expressive Softgoal Conceptualization for Quality Requirements Analysis. In: Conceptual Modeling - ER, 25th International Conference on Conceptual Modeling, Arizona, (2006). 